\title{
ON SMALL PERIOD, LARGE AMPLITUDE NORMAL MODES OF NATURAL HAMILTONIAN SYSTEMS
}

\author{
E. W. C. VAN GroeseN* \\ Mathematical Institute, University of Nÿmegen, Toernooiveld, Nÿmegen, The Netherlands
}

(Received 20 October 1984; received for publication 9 April 1985)

Key words and phrases: Periodic solution, Hamiltonian system, variational methods, natural constraints.

\section{INTRODUCTION}

IN THIS paper we shall consider periodic motions of a system described by a set of second order autonomous equations

$$
-\ddot{x}=V^{\prime}(x)
$$

for the $N$-vector function $x(t)$, where $V$ is a potential energy function defined on $\mathbb{R}^{x}$. The system described by (1) is a Hamiltonian system for which the Hamiltonian

$$
H(x, p)=\frac{1}{2} p \cdot p+V(x), \quad(x, p) \in \mathbb{R}^{N} \times \mathbb{R}^{v},
$$

being the sum of kinetic energy and potential energy, is a so-called natural Hamiltonian.

Our investigation of (1) will concentrate on finding and characterizing periodic solutions which have an (arbitrarily) prescribed value of the period. More specifically, we shall assume that $V$ satisfies the following condition

$$
\text { (V0) } V \in C^{2}\left(\mathbb{R}^{N}, R\right), \quad V(0)=0 \quad \text { and } \quad V \text { is even: } V(x)=V(-x) \text { for } x \in \mathbb{R}^{v} \text {. }
$$

Berger (see e.g. [2]) has noted that this evenness assumption makes it possible to look for periodic solutions which are odd, thereby reducing the problem to one with homogeneous Dirichlet boundary conditions. In this paper, we shall look for an even more special kind of periodic solution, to be called normal modes (see [9] and the references therein for a motivation of this terminology). These are periodic solutions of $(1)$ for which the trajectory in $\mathbb{R}^{N}$ is a symmetric curve through the origin along which the motion oscillates back and forth between the two endpoints of the curve, which are rest points for the motion. These normal modes are completely characterized by a solution of a boundary value problem (see e.g. [6]). In fact, let $x$ be for some $\lambda>0$ a solution of

$$
\begin{aligned}
-\ddot{x} & =\lambda V^{\prime}(x), \quad t \in(0,1) \\
x(0) & =\dot{x}(1)=0,
\end{aligned}
$$

and define a continuation map $\mathscr{b}$ as

$$
\mathscr{G} x(t):=\text { odd continuation of the function } \bar{x}(t)=\left\{\begin{array}{ll}
x(t) & \text { for } t \in[0,1] \\
x(2-t) & \text { for } t \in[1,2]
\end{array}\right. \text {. }
$$

* Sponsored by the United States Army under Contract No. DAAG29-80-C-0041. This research has been partially supported by the Niels Stensen Stichting, Amsterdam.

$\dagger$ Curtent address: Department of Applied Mathematics, Twente University of Technology, P.O. Box 217, Enschede, The Netherlands. 
Then the normal mode corresponding to a solution $x$ of (2) is given by the function

$$
\hat{x}(t):=\operatorname{Ex}\left(t / \vee \lambda_{i}\right)
$$

which is easily verified to be a smooth solution of (1) on all of $R$ which has period $T=4 V \lambda$. Note that this normal mode will have $T$ as its minimal period iff the solution of (2) satisfies $\dot{x}(t) \neq 0$ for $t \in[0,1)$.

Simple examples show that without further conditions on $V$ no periodic solution with arbitrary period will exist in general. Therefore consider the following (super quadratic) growth condition:

(V1) There exist numbers $\mu>2$ and $R>0$ such that

$$
V^{\prime}(x) \cdot x \geqslant \mu V(x)>0 \text { for all } x \in \mathbf{P}^{*},|x| \geqslant R \text {. }
$$

Requiring only condition (V1), Rabinowitz [14] (see also $[13,15]$ showed that for any $T>0$ and any $A>0$ there exists a solution of (1) which is periodic with period $T$ and which has sup norm larger than $A$. A simplified proof of this result for even or convex potentials is contained in [8]. In both cases nothing can be said about the minimal period of such a solution.

Ambrosetti and Mancini [1] obtained the existence of at least one solution with prescribed minimal period for a restricted class of (noneven) potentials $V$ by minimizing the dual action functional on a naturally constrained set. Here we shall apply this device of Nehari [11] to the action functional itself. For even potentials our conditions are somewhat less stringent than those in [1]. Moreover, under additional conditions we shall derive a multiplicity result for $N$ solutions, all having minimal period.

In this paper we shall require an additional condition which is essentially a monotonicity condition for the growth of $V$ on all of any ray through the origin:

$$
V(x)>0 \text { and } V^{\prime \prime}(x) \cdot x \cdot x-V^{\prime \prime}(x) \cdot x>0 \text { for all } x \in \mathbb{R}^{N \backslash\{0\} .}
$$

Assuming (V0)-(V2) to be satisfied it will be shown in Section 2 that for any $T>0$ less than the smallest nontrivial period of the linearized system (if nontrivial, else for all $T$ ) there exists a normal mode $\hat{x}_{T}$ which has minimal period $T$, and that $\left|\hat{x}_{T}\right|_{C^{0}} \rightarrow \infty$ as $T \rightarrow 0$.

In [14] and [8] the periodic solutions were found using topological mini-max methods for the action functional on the set of $T$-periodic functions in [14] and on certain naturally constrained subsets in [8]. Here we shall show that it is possible to define a naturally constrained set $\hat{v}_{T}$ such that the normal modes $\hat{x}_{T}$ are characterized as the elements for which the action functional is minimized on this set $\hat{N}_{T}$. For the solution of the boundary value problem (2) with $\lambda=(T / 4)^{2}$ which defines this normal mode via (4) this naturally constrained minimization problem reads

$$
\inf \left\{\int_{0}^{1}\left\{\frac{1}{2} \dot{x}^{2}-\lambda V(x)\right\}: x \neq 0, x(0)=0, \int_{0}^{1}\left\{\dot{x}^{2}-\lambda V^{\prime}(x) \cdot x\right\}=0\right\} .
$$

A natural constraint of this type has been used for a special class of scalar equations for the first time by Nehari [11].

In Section 3 we shall consider potentials $V$ which have a direction of maximum increase, i.e. for which there exists a vector $e \in S^{N-1}$, the unit sphere in $\mathbb{R}^{N}$, such that on each sphere with radius $r>0, V$ attains its maximum value at the point $r e$. For such potentials, which include the class of similarity and, in particular, homogeneous potentials, it is shown that the 
solution of the naturally constrained minimization problem (5) provides a similar normal mode, i.e. a normal mode which has a trajectory that lies on the ray through the origin in the direction $e$.

In Section + we shall consider some multiplicity results for normal modes with minimal period $T$. For any similarity potential the existence of $N$ similar normal modes in different directions of "stationary" increase of $V$ will be proven. In case $V$ is not a similarity potential, a condition will be given that guarantees the existence of at least $N$ distinct normal modes with minimal period $T$. This condition, unlike (V1), (V2), which are conditions on the growth of $V$ on rays through the origin, is a condition on the geometry of the level sets of $V$. A condition of this kind has been used by Ekeland and Lasry [5] to prove the existence of at least $N$ distinct periodic solutions of the same prescribed energy (instead of prescribed period) and precisely the same condition provides the existence of at least $N$ normal modes of (1) with prescribed energy, see [7], or with prescribed normalized potential energy (i.e. $\int_{0}^{1} V(x)$ ), see [9]. The result to be obtained in Section 4 , theorem 4.3 , seems to be the first multiplicity result for solutions with minimal period for superquadratic potentials (for the subquadratic case, see $[3,8])$.

\section{THE NATURALLY CONSTRAINED MINIMIZATION PROBLEM}

In this section the naturally constrained minimization problem will be introduced in a precise way and is used to obtain the existence of normal modes of prescribed, small, minimal period.

We start to introduce some notation and recall some standard results that are needed further on. Let $H_{1}:=H_{1}\left([0,1], \mathbb{R}^{k}\right)$ be the Sobolev space of $N$-vector functions $x:[0,1] \rightarrow \beta^{\prime \prime}$ which are, together with their generalized first derivative, square integrable. $H_{1}$ is a Hilbert space which is continuously embedded in the set $C^{0}:=C_{0}\left([0,1], \mathbb{R}^{N}\right)$ of continuous vector functions. Therefore, the set

$$
E:=\left\{x \in H_{1}: x(0)=0\right\}
$$

is neatly defined and, being a subspace, $E$ is a Hilbert space itself. Since

$$
\int \dot{x}^{2} \geqslant\left(\frac{\pi}{2}\right)^{2} \int x^{2}
$$

where here and in the following, $\int$ denotes integration with respect to $t$ over $(0,1)$, we can, and shall, take \|\| defined by $\|x\|:=\left\{\int \dot{x}^{2}\right\}^{1 / 2}$ as a norm on $E$ (equivalent to the usual $H_{1}$-norm). With $\mid C_{C^{0}}$ the norm of $C^{0},|x|_{C^{0}}:=\max _{t \in[0.1]}|x(t)|$, we also have

$$
|x|_{c^{0}} \leqslant\|x\| \text { for all } x \in E \text {. }
$$

Moreover, $E$ is compactly embedded in $C^{0}$ : any sequence $\left\{x_{n}\right\} \subset E$ with $\left\|x_{n}\right\|$ uniformly bounded, has a subsequence $\left\{x_{n}\right\}$ which converges weakly in $E$ and strongly in $C^{0}$.

For any $\lambda>0$ define the functional $\psi_{\lambda}$ on $E$ by

$$
\psi_{\lambda}(x):=\int\left\{\frac{1}{2} \dot{x}^{2}-\lambda V(x)\right\}, \quad x \in E
$$

Note that $\psi_{\lambda}$ is well defined, is not bounded from above and is twice differentiable (if $\left.V \in C^{2}\left(\mathbb{R}^{N}\right)\right)$ on $E$. The first result states that looking for critical points of $\psi_{i}$ on $E$ is a 
variational formulation of the problem of finding solutions of (2), but that, in the case we are considering, any nontrivial solution of (2) is not simply a (local) minimum of $\psi_{i}$ on $E$.

Proposition 2.1. Assume $V \in C^{2}\left(\mathbb{R}^{N}\right)$ and let $\lambda>0$. Then any function $x \in E$ is a classical solution of the boundary value problem (2) iff $x$ is a critical point of $\psi_{\lambda}$ on $E$. Furthermore, if $V$ satisfies (V1) then $\psi_{\lambda}$ is not bounded from below on $E$ and if $V$ satisfies (V2) then any nonzero critical point of $\psi_{\lambda}$ is not a local minimum of $\psi_{\lambda}$ on $E$.

Proof. The first statement is a standard result from the classical calculus of variations. Note in particular that the condition $\dot{x}(1)=0$ results as a natural boundary condition from the variational formulation. Next, let $S$ denote the unit sphere in $E: S:=\{x \in E:\|x\|=1\}$, and consider for fixed $y \in S$ the function $g: \mathbb{R}_{+} \rightarrow \mathbb{R}$ defined by

$$
g(\rho):=\psi_{\lambda}(\rho y), \quad \rho>0 .
$$

We shall show that as a consequence of (V1), $g(\rho) \rightarrow-\infty$ as $\rho \rightarrow \infty$, which implies that $\psi_{i}$ is not bounded from below on $E$. To that end, observe that by integrating the inequality in (V1) it follows that for some constants $a>0$ and $b \leq 0$ :

$$
V(x) \geqslant a|x|^{\mu}+b \text { for all } x \in \mathbb{R}^{v} \text {. }
$$

Therefore

$$
\psi_{\lambda}(\rho y)=\frac{1}{2} \rho^{2}-\lambda \int V(\rho y) \leqslant \frac{1}{2} \rho^{2}-a \rho^{\mu} \int|y|^{\mu}-b,
$$

and, since $\mu>2$, the result follows. Finally, consider the second variation of $\psi$; at $x$ in the direction $\xi \in E$, given by

$$
Q_{\dot{\lambda}}(x ; \xi)=\int\left(\dot{\xi}^{2}-\lambda V^{\prime \prime}(x) \xi \cdot \xi\right\}
$$

Then, for $x \equiv 0$, condition (V2) immediately implies that $Q_{\lambda}(x: \varepsilon x)$ is negative for all $\varepsilon \in \mathbb{R} \backslash\{0\}$ if $x$ is a solution of (2), which shows that $x$ is not a local minimum of $\psi_{\lambda}$ on $E$.

From now on we shall assume that $V$ satisfies (V0)-(V2). In order to explain the introduction of the natural constraint $v_{\lambda}$ below, consider for fixed $y \in S$ the function $g$ defined by (2.4). The behaviour of $g$ at 0 and at $x$ is known and independent of $y \in S$ and $\lambda>0$

$$
g(0)=0 \text { and } g(\rho) \rightarrow-\infty \text { as } \rho \rightarrow x \text {, }
$$

but for finite values of $\rho$ the behaviour of $g$ depends on the detailed behaviour of $V$ and on the choice of $y \in S$ and $\lambda>0$. However, condition (V2) implies another global property of $g$, independent of $y \in S$ and of $\lambda$ if $\lambda>0$ is sufficiently small. To investigate this, consider the first and second derivative of $g$. At any critical point $\hat{\rho}>0$ of $g, g^{\prime}(\hat{\rho})=0$, the second derivative is given by

$$
g^{\prime \prime}(\hat{\rho})=\lambda \hat{\rho}^{-2} \int\left\{V^{\prime}(\hat{\rho} y) \cdot \hat{\rho} y-V^{\prime \prime}(\hat{\rho} y) \hat{\rho} y \cdot \hat{\rho} y\right\}
$$

Hence $g^{\prime \prime}(\hat{\rho})<0$ because of (V2), which implies that if $g$ has a critical point $\hat{\rho}>0$. then $\hat{\rho}$ is the unique positive critical point of $g$ and $g$ attains its global maximum at $\hat{\rho}$. We shall now 
show that for $\lambda>0$ sufficiently small and any $y \in S, g$ has indeed a positive critical point. Given (2.5), it suffices to show that there exists $\lambda^{*}>0$ such that for any $\lambda \in\left(0, \lambda^{*}\right) g$ is positive for $\rho>0$ sufficiently small. Since $g \in C^{2}$, with $g(0)=g^{\prime}(0)=0$, this will be the case if $g^{\prime \prime}(0)>$ 0 . Define

$$
T^{*}:=4 \sqrt{ } \lambda^{*}, \quad \text { and } \quad \lambda^{*}:=\left\{\begin{array}{lll}
\infty & \text { if } V^{\prime \prime}(0) \equiv 0 \\
\left(\frac{\pi}{2}\right)^{2} \sigma^{-1} & \text { if } \quad V^{\prime \prime}(0) \neq 0
\end{array}\right. \text {, }
$$

where $\sigma$ is the largest (positive) eigenvalue of $V^{\prime \prime}(0)$. Note that, in case $V^{\prime \prime}(0) \neq 0, T^{*}$ is the smallest (nontrivial) period of the solutions of the linearized system

$$
-\ddot{x}=V^{\prime \prime}(0) x \text {. }
$$

Using inequality (2.1) in the expression for $g^{\prime \prime}(0)$, it follows that

$$
g^{\prime \prime}(0)=\int\left\{\dot{y}^{2}-\lambda V^{\prime \prime}(0) y \cdot y\right\} \geqslant \int\left\{\left(\frac{\pi}{2}\right)^{2} I_{N}-\lambda V^{\prime \prime}(0)\right\} y \cdot y>0 \quad \text { for } \quad \lambda \in\left(0, \lambda^{*}\right)
$$

where $I_{N}$ is the identity matrix in $\mathbb{R}^{N}$. Hence, for $\lambda \in\left(0, \lambda^{*}\right), g$ has for any $y \in S$ a unique, positive critical point at which $g$ is maximal.

Now define the set $\dot{N}_{i}$ as

$$
N_{\lambda}:=\left\{x \in E: x \not \equiv 0, \int \dot{x}^{2}=\lambda \int V^{\prime}(x) \cdot x\right\} .
$$

In view of the foregoing, for $\lambda \in\left(0, \lambda^{*}\right), \mathcal{N}_{\lambda}$ can also be described as

$$
\mathcal{N}_{\lambda}=\left\{\hat{\rho} y: y \in S, \hat{\rho} \text { is the unique solution of } \max _{\rho>0} \psi_{\lambda}(\rho y)\right\} .
$$

By taking the $L_{2}$-innerproduct of the equation in (2) with the function $x$, it is clear that any nontrivial critical point of $\psi_{\lambda}$ on $E$ belongs to $\mathcal{N}_{\lambda}$. The next result states that it is also true that any critical point of the restriction of $\psi_{\lambda}$ to $\mathcal{N}_{i}$ is a critical point of $\psi$ on $E$. (For a special class of scalar equations, a constraint like (2.7) has been used for the first time by Nehari [11].)

Proposition 2.2. Let $V$ satisfy $\left(V_{0}\right)-\left(V_{2}\right)$ and let $\lambda \in\left(0, \lambda^{*}\right)$, where $\lambda^{*}$ is defined in (2.6). Then the set $\dot{\lambda}_{\lambda}$ given by (2.7) is a smooth, symmetric manifold in $E$ which has codimension 1. Moreover, $\hat{\mathcal{N}}_{\lambda}$ is a natural constraint for the functional $\psi_{\lambda}$ on $E$, i.e. any critical point of the restriction of $\psi_{i}$ to $\gamma_{\lambda}$ is a solution of the boundary value problem (2).

Proof. Define a functional $\phi_{\lambda}$ on $E$ by

$$
\phi_{\lambda}(x):=\int\left\{\dot{x}^{2}-\lambda V^{\prime}(x) \cdot x\right\}
$$

Then $\phi_{\lambda}$ is differentiable on $E$ and the set $\mathcal{N}_{\lambda}$ is given by $\mathcal{N}_{\lambda}=\left\{x \in E: \phi_{\lambda}(x)=0\right\} \backslash\{0\}$. For $x \in \mathcal{N}_{\lambda}$ it is readily seen that $\left\langle\phi_{\lambda}^{\prime}(x), x\right\rangle=\lambda \int\left\{V^{\prime}(x) \cdot x-V^{\prime \prime}(x) x \cdot x\right\}<0$, from which it follows that $\phi_{\lambda}^{\prime}(x) \neq 0$. Hence, at every $x \in \mathcal{N}_{\lambda}$, the tangent space to $\mathcal{N}_{\lambda}$ at $x$ has codimension 1 . Next we show that $x \equiv 0$ is an isolated point of the set $\left\{x \in E: \phi_{\lambda}(x)=0\right\}$, which will imply that $\mathcal{N}_{\lambda}$ 
is smooth. To that end, let $m>0$ be any number if $V^{\prime \prime}(0) \equiv 0$, and let $m>\sigma$ if $\sigma$ is the largest eigenvalue of $V^{\prime \prime}(0) \neq 0$. Then there exists a $\rho>0$, depending only on $m$, such that

$$
V^{\prime}(x) \cdot x \leqslant m x^{2} \quad \text { for all } x \in \mathbb{R}^{v}, \quad|x| \leqslant \rho,
$$

and $\rho(m) \rightarrow 0$ as $m \downarrow \sigma$. With inequalities (2.1) and (2.2), it follows that if $x \in \wedge_{i}$ and $\|x\| \leqslant \rho$, then

$$
\left(\frac{\pi}{2}\right)^{2} \int x^{2} \leqslant \int \dot{x}^{2}=\lambda \int V^{\prime}(x) \cdot x \leqslant \lambda m \int x^{2},
$$

so that $x \in \dot{N}_{i}$ must satisfy $\|x\|>\rho$ if $\lambda<(\pi / 2)^{2} \cdot m^{-1}$. In case $V^{\prime \prime}(0) \neq 0, \rho \rightarrow 0$ as $\lambda \uparrow \dot{\lambda}^{*}$. but, for future reference, note that for any $\varepsilon>0$ there exists a number $\rho_{0}$. such that

$$
\mathfrak{N}_{i} \cap\left\{x \in E:\|x\| \leqslant \rho_{0}\right\}=\varnothing \text { for } \lambda \in\left(0, \lambda^{*}-\varepsilon\right] \text {. }
$$

Finally, to show that $\nu_{\lambda}$ is a natural constraint, the multiplier rule states that a critical point $x$ of $\psi_{\lambda}$ on $\hat{\gamma}_{i}$ satisfies for some number $\nu \in \mathbb{R}$ the equation

$$
-\ddot{x}-\lambda V^{\prime}(x)=\nu\left\{-2 \ddot{x}-\lambda V^{\prime}(x)-\lambda V^{\prime \prime}(x) x\right\},
$$

and the boundary condition $x(0)=\dot{x}(1)=0$. Multiplying this equation by $x$ and integrating over $(0,1)$ readily shows that $\nu=0$ because $x \in N_{\lambda}$ and $V$ satisfies (V2). Hence, any critical point of $\psi_{\lambda}$ on $N_{\lambda}$ satisfies (2), which completes the proof.

We are now in a position to define for given $\lambda>0$ the naturally constrained minimization problem, the value of which will be denoted by $c(\lambda)$ :

$$
c(\lambda)=\inf \left\{\psi_{\lambda}(x): x \in \dot{N}_{\lambda}\right\}
$$

Remark 2.3. In view of the characterization $(2.8)$ for $\hat{N}_{i}$, it is easily seen that for $i \in\left(0, \lambda^{*}\right)$ (2.11) is an explicit minimization formulation of a mini-max problem for $\psi_{\lambda}$ on all of $E$ :

$$
c(\lambda)=\inf _{y \in s} \max _{\rho>0} \psi_{\lambda}(\rho y)
$$

This clearly demonstrates the role of the natural constraint $\dot{V}_{i}$ in characterizing some of the critical points of $\psi_{\lambda}$ which are of saddle point type on $E$ as constrained minima for $\psi_{\lambda}$ on $\hat{N}_{i}$.

Proposition 2.4. Let $V$ satisfy (V0)-(V2) and let $\lambda^{*}$ be defined as in (2.6). Then $c(\lambda)$ is finite and positive for all $\lambda \in\left(0, \lambda^{*}\right)$ and $c$ is monotonically decreasing on $\left(0, \lambda^{*}\right)$. Furthermore, for each $\lambda \in\left(0, \lambda^{*}\right)$, the minimization problem (2.11) has a solution $x_{\lambda}$, and $\left|x_{\lambda}\right|_{C^{0}} \rightarrow x$ as $\lambda \rightarrow 0$.

Moreover, any minimizing sequence for (2.11) has a subsequence which converges in \|\| norm, and hence uniformly on $[0,1]$, to a solution of $(2.11)$.

Before giving its proof, let us use proposition 2.4 to obtain the existence of small period, large amplitude normal modes for equation (1).

THEOREM 2.5. Let $V$ satisfy (V0)-(V2) and let $T^{*}$ be given by (2.6). Then, for any $T \in\left(0, T^{*}\right)$ equation (1) has a solution $\hat{x}_{T}$ which is a normal mode with minimal period $T$. Moreover, $\left|\hat{x}_{T}\right|_{c^{0} \rightarrow x}$ as $T \rightarrow 0$, and $\hat{x}_{T}$ corresponds via the transformation (4) to a solution $x_{j}$ of the naturally constrained minimization problem (2.11) for $\lambda=(T / 4)^{2}$. 
Proof. Let $\hat{x}_{T}$ denote the normal mode which corresponds via the transformation (4) to a solution $x_{i}$ of (2.11). Since $\left|\hat{x}_{T}\right|_{C^{0}}=\left|x_{i}\right|_{C^{0}}$, it only remains to be shown that $\hat{x}_{T}$ has minimal period. Therefore, suppose that $\bar{x}$ is any solution of (2) for which the corresponding normal mode does not have minimal period. Then there exists some integer $k \in \mathbb{N}, k \geqslant 3$ and $k$ is odd, such that the function $y$ defined by $y(t):=x(t / k), t \in[0,1]$, satisfies $y(0)=\dot{y}(1)=0$ (because $k$ is odd) and the equation in (2) with $\lambda$ replaced by $\lambda / k^{3}$. Hence $y \in r_{\lambda k} z$ and by the definition of the function $c: c\left(\lambda / k^{2}\right) \leqslant \psi_{\lambda / k} 2(y)$. As is easily seen $\psi_{i k} 2(y)=$ $\left(1 / k^{2}\right) \psi_{\lambda}(\vec{x})$, from which it follows that

$$
c\left(\lambda / k^{2}\right) \leqslant \frac{1}{k^{2}} \psi_{\lambda}(\bar{x})
$$

Consequently, if $\bar{x}$ were a solution of the minimization problem (2.11), it would follow that $c\left(\lambda / k^{2}\right) \leqslant\left(1 / k^{2}\right) c(\lambda)$, in contradiction with the fact that the function $c$ is monotonically decreasing. This shows that $\hat{x}_{T}$ has a minimal period $T$.

Proof of proposition 2.4. On the set $\mathcal{N}_{\lambda}$ the functional $\psi_{\lambda}$ can be written as

$$
\psi_{i}(x)=\lambda \int W(x)
$$

where $W(x)=(1 / 2) V^{\prime}(x) \cdot x-V(x)$. Because of condition (V2), $W$ satisfies $W^{\prime}(x) \cdot x>0$ which implies that for any $x \neq 0$ the function $\rho \rightarrow W(\rho x)$ is monotonically increasing for $\rho>0$, and hence $W(x)>W(0)=0$ for $x \neq 0$. This shows that $c(\lambda) \geqslant 0$. That $c(\lambda)>0$ follows as soon as the existence of a solution has been proved because $0 \notin \mathcal{N}_{\lambda}$. To show that $c$ is monotone, let $0<\lambda_{1}<\lambda_{2}<\lambda^{*}$, and let $x_{1}(\equiv 0)$ be a solution of (2.11) for $\lambda=\lambda_{1}$. Then $x_{1} \in \hat{\nu}_{\lambda_{1}}$ and for some $\rho \neq 1, \rho>0, \rho x_{1} \in \mathcal{N}_{i_{2}}$. Because of (2.8) it follows that $\psi_{\lambda_{1}}\left(\rho x_{1}\right)<\psi_{\lambda_{1}}\left(x_{1}\right)$. Since $\psi_{\lambda_{1}}(x)>\psi_{\lambda_{2}}(x)$ for all $x \neq 0$, the result follows easily: $c\left(\lambda_{1}\right)=\psi_{i_{1}}\left(x_{1}\right)>\psi_{i_{1}}\left(\rho x_{1}\right)>$ $\psi_{\lambda_{2}}\left(\rho x_{1}\right) \geqslant c\left(\lambda_{2}\right)$. Next, let $x_{\lambda}$ be a solution of (2.11). To show that $\left|x_{i}\right| c^{0} \rightarrow x$ as $\lambda \rightarrow 0$, suppose, on the contrary, that $\left|x_{\lambda}\right|_{C^{0}}$ is uniformly bounded. Then $\int V^{\prime}\left(x_{\dot{\lambda}}\right) \cdot x_{\dot{\lambda}}$ is uniformly bounded and since $x_{\lambda} \in \hat{\gamma}_{i}$ it follows that $\left\|x_{\lambda}\right\| \rightarrow 0$ as $\lambda \rightarrow 0$, contradicting property (2.10).

It remains to prove the existence of a solution of (2.11). First observe that as a consequence of condition (V1), for some $a \in \mathbb{R}$

$$
\psi_{\lambda}(x) \geqslant \int\left\{\frac{1}{2} \dot{x}^{2}-\frac{\lambda}{\mu} V^{\prime}(x) \cdot x\right\}+a=\left(\frac{1}{2}-\frac{1}{\mu}\right) \int \dot{x}^{2}+a \text { for } x \in \dot{\nu}_{\lambda} .
$$

Now, let $\left\{x_{n}\right\}$ be any minimizing sequence, i.e. $x_{n} \in \hat{\gamma}_{i}$ for all $n \in \mathbb{N}$ and $\psi_{\lambda}\left(x_{n}\right) \rightarrow c(\lambda)$ as $n \rightarrow x$. Since $\mu>2$, it follows from (2.15) that $\left\|x_{n}\right\|$ is uniformly bounded. Hence, some subsequence, again to be denoted by $\left\{x_{n}\right\}$, converges weakly in $E$ and in $C^{0}$ to some $\hat{x} \in E$. We shall show that $\hat{x}$ is a solution of the minimization problem (2.11).

Since the norm functional is lower semicontinuous with respect to weak convergence, $\|\hat{x}\| \leqslant \lim$ inf $\left\|x_{n}\right\|$, and since $\left\{x_{n}\right\}$ converges in $C^{0}, \int V\left(x_{n}\right) \rightarrow \int V(\hat{x})$ and $\int V^{\prime}\left(x_{n}\right)$ $\cdot x_{n} \rightarrow \int V^{\prime}(\hat{x}) \cdot \hat{x}$ as $n \rightarrow x$. Consequently $\psi_{\lambda}(\hat{x}) \leqslant c(\lambda)=\lim \psi_{\lambda}\left(x_{n}\right)$ and $\phi_{\dot{\lambda}}(\hat{x}) \leqslant 0$ where $\phi_{\lambda}$ is defined by (2.9). We shall show that $\hat{x} \neq 0$ and that $\phi_{\lambda}(\hat{x})=0$, i.e. that $\hat{x} \in \hat{\gamma}_{\lambda}$. Indeed, that $\hat{x} \neq 0$ follows since $x_{n} \in \gamma_{\lambda}$ and $x_{n} \rightarrow 0$ in $C^{0}$ would imply $\left\|x_{n}\right\| \rightarrow 0$, contradicting the fact that 0 is an isolated point of $\hat{v}_{i}$. Furthermore, if $\phi(\hat{x})<0$, then there exists a $\rho>0$ such that $\phi(\rho \hat{x})=0$, and in fact $\rho<1$ because of the behaviour of the function $\rho \rightarrow \psi_{\lambda}(\rho x)$. Then, by 
(2.14) and the monotonicity of $W$ :

$$
\psi_{\lambda}(\rho \hat{x})=\lambda \int W(\rho \hat{x})<\lambda \int W(\hat{x})=\lim \lambda \int W\left(x_{n}\right)=\lim \psi_{\lambda}\left(x_{n}\right)=c(\lambda),
$$

whereas, by definition of $c(\lambda): \psi_{\lambda}(\rho \hat{x}) \geqslant c(\lambda)$. This contradiction implies that $\phi(\hat{x})=0$. From this it follows that $\left\|x_{n}\right\| \rightarrow\|\hat{x}\|$, which, together with the fact that $x_{n}$ converges weakly to $\hat{x}$, implies that $x_{n}$ converges strongly in $\hat{E}:\left\|x_{n}-\hat{x}\right\| \rightarrow 0$ as $n \rightarrow \infty$. From these results, it is immediate that $\hat{x}$ is a solution of (2.11) since $\psi_{\lambda}\left(x_{n}\right) \rightarrow \psi_{\lambda}(\hat{x})$ as $n \rightarrow \infty$ and thus $\psi_{\lambda}(\hat{x})=c(\lambda)$. This completes the proof.

\section{SIMILAR NORMAL MODES}

The simplest normal modes are those of which the trajectory in $\mathbb{R}^{N}$ is on a straight line through the origin. Such periodic solutions are called similar normal modes (cf. [16]). Stated in another way, a similar normal mode in the direction $e \in S^{v-1}:=\left\{e \in \mathbb{R}^{N}:|e|=1\right\}$ is related via (4) to a solution $x$ of the boundary value problem (2) which is of the form

$$
x(t)=\alpha(t) e,
$$

where $\alpha(t)$ is a scalar function. In order that (3.1) is a solution of (2), the vector $e$ has to satisfy

$$
V^{\prime}(\alpha e)=\left(V^{\prime}(\alpha e) \cdot e\right) e
$$

and $\alpha$ has to be a solution of the scalar equation

$$
-\ddot{\alpha}=\lambda V^{\prime}(\alpha e) \cdot e, \quad \alpha(0)=\dot{\alpha}(1)=0 .
$$

Note that the corresponding normal mode has minimal period iff $\dot{\alpha}(t) \neq 0$ for $t \in(0,1)$, i.e. iff $\alpha$ is monotonically increasing (or decreasing) on $[0,1]$.

For general potential $V$ there will be no direction $e \in S^{N-1}$ such that (3.2) is satisfied for all $\alpha>0$. If $V$ happens to have such a direction, as is for instance the case if $V$ is a similarity potential (see Section 4). conditions (V0)-(V2) will guarantee that (3.3) has a solution, as we shall see.

Definition 3.1. V is said to have a direction of stationary increase $e \in S^{n-1}$ if for each $r>0$, re is a critical point of the function $V$ on the sphere $r S^{N-1}$. $V$ is said to have a direction of maximal increase $e \in S^{n-1}$ if for each $r>0$ :

$$
V(r e)=\max _{x \in r S^{n-1}} V(x)
$$

As is easily verified, $V$ has a direction of stationary increase $e \in S^{N-1}$ iff (3.2) is satisfied for all $\alpha>0$, and if $e$ is a direction of maximal increase, then $e$ is a direction of stationary increase.

For $e \in S^{N-1}$ consider the set $E^{1}:=\{\alpha:[0,1] \rightarrow \mathbb{R}: \alpha e \in E\}$, which is nothing but the usual space of scalar $H_{1}$-functions $\alpha$ with $\alpha(0)=0$. In a natural way, the restriction of the functional $\psi_{\lambda}$ and the set $\mathcal{N}_{\lambda}$ to functions $\alpha e \in E$ leads to the functional

$$
\psi_{\lambda, e}(\alpha):=\psi_{\lambda}(\alpha e), \quad \alpha \in E^{1}
$$


and to the set

$$
\therefore \text { i.e }:=\left\{\alpha \in E^{1}: \alpha e \in V_{j}\right\} \text {. }
$$

We shall consider the minimization problem

$$
\inf \left\{\psi_{i, e}(\alpha): \alpha \in S_{\lambda . e}\right\} \text {. }
$$

This minimization problem is for $N=1$ of the form considered in section 2, (2.11), with a potential $V_{e}$ defined by

$$
V_{e}(\alpha):=V(\alpha e), \quad \alpha \in \mathbb{R},
$$

and the results of that section may be applied.

Proposition 3.2. Let $V$ satisfy (V0)-(V2) and let $T^{*}$ be defined by (2.6). If $e \equiv S^{\vee-1}$ is any direction of stationary increase for $V$, then for every $T \in\left(0, T^{*}\right)$ there exists a similar normal mode $\hat{x}_{T}$ in the direction $e$ which has minimal period $T$, and $\left|\hat{x}_{T}\right|_{C} \rightarrow x$ as $T \rightarrow 0$. This similar normal mode is related via (4) to a solution $x_{;}$, of (2), where $x_{i}=\alpha_{i}$ e and $\alpha_{\dot{i}}$ is a solution of the minimization problem (3.7) for $\lambda=(T / 4)^{2}$.

Proof. We investigate the minimization problem (3.7). Since $V_{e}^{\prime}(\alpha)=V^{\prime \prime}(a e) \cdot e$ and $V_{e}^{\prime \prime}(\alpha)=V^{\prime \prime}(\alpha e) e \cdot e$, it is easily verified that the potential $V_{e}$ satisfies conditions (V0)-(V2) because $V$ does. Next we define the values $\lambda^{*}$ and $T^{*}$ for problem (3.7):

$$
T_{e}^{*}:=4 V\left(\lambda_{e}^{*}\right), \quad \lambda_{e}^{*}:= \begin{cases}x & \text { if } \quad V_{e}^{\prime \prime}(0)=0 \\ \left(\frac{\pi}{2}\right)^{2}\left[V_{e}^{\prime \prime}(0)\right]^{-1} & \text { if } \quad V_{e}^{\prime \prime}(0) \neq 0\end{cases}
$$

Since $V_{e}^{\prime \prime}(0)=V^{\prime \prime}(0) e \cdot e$, it is clear that $\lambda_{e}^{*} \geqslant \lambda^{*}$. Consequently, for any $T \equiv\left(0, T^{*}\right) \subset$ $\left(0, T_{e}^{*}\right)$ we can apply the results of proposition 2.4 and theorem 2.5 for the special case $N=$ 1 and potential $V_{e}$. In this way we find a solution $\alpha_{j}$ of (3.7) to which there corresponds a normal mode with minimal period $T$. Since $\alpha_{i}$ satisfies the boundary value problem (2) for $V_{e}$, $\alpha_{j}$ is a solution of (3.3). Since $e$ is a direction of stationary increase, (3.2) is satisfied, so that $\alpha_{i} e$ defines via the transformation (4) a normal mode $\hat{x}_{T}$ in the direction $e$ with minimal period $T$. Moreover, since $\mid \alpha ; c^{0} \rightarrow x$ as $\lambda \rightarrow 0$, it follows that $\left|\hat{x}_{T}\right| c^{0} \rightarrow x$ as $T \rightarrow 0$.

Remark 3.3. The result of proposition 3.2 can also be stated as follows: If $e$ is a direction of stationary increase for $V$, the functional $\psi_{;}$attains its finite, minimum value on the set

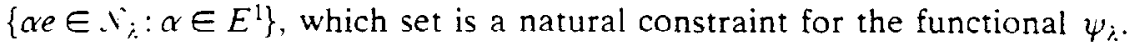

The next result characterizes the solution set of the naturally constrained minimization problem (2.11) in case $V$ has a direction of maximal increase.

Proposition 3.4. Let $V$ satisfy (V0)-(V2) and let $\lambda \in\left(0, \lambda^{*}\right)$ where $\lambda^{*}$ is given by (2.6). Assume, moreover, that $V$ has a direction of maximal increase. Then the solution set of the minimization problem (2.11) consists of all the functions $x_{i}=\alpha_{i, e}$ where $e$ is any direction of maximal increase and $\alpha_{i}$ is any solution of (3.7). 
Proof. Let $e \in S^{l-1}$ be a direction of maximal increase and let $x$ be a solution of $(2.11)$. We consider the "normalized" radial projection $p$ of $x$ onto the direction $e: p(t):=\rho x(t) \mid e$, where $\rho>0$ is chosen in such a way that $p \in i_{i}$. Then

$$
\dot{p}=\rho\left(\frac{\dot{x} \cdot x}{|x|}\right) e \text { and } \int \dot{p}^{2} \leqslant \rho^{2} \int \dot{x}^{2}
$$

with equality only if $x(t)$ and $\dot{x}(t)$ are parallel for all $t \in[0,1]$, i.e. only if $x(t)=\beta(t) \tilde{e}$ for some $\bar{e} \in S^{v-1}$ and some scalar function $\beta$. Since $e$ is a direction of maximal increase, it holds $V(p(t)) \geqslant V(\rho x(t))$, so that

$$
\psi_{\dot{\lambda}}(p) \leqslant \frac{1}{2} \rho^{2} \int \dot{x}^{2}-\lambda \int V(\rho x)=\psi_{i}(\rho x) \leqslant \psi_{\lambda}(x)
$$

where the last inequality follows since $x \in N$ and because of the characterization (2.8), which also shows that it is an equality iff $\rho=1$. Since $x$ is a solution of (2.11), and $p \in N$, we also have $\psi_{i}(x) \leqslant \psi_{i}(p)$. Therefore it follows that $\rho=1$, that $x(t)=|x(t)| \vec{e}$ and that $V(|x(t)| \tilde{e})=$ $V(|x(t)| e)$. This shows that $\dot{e}$ must be a direction of maximal increase for $V$, and the result of the proposition follows easily.

\section{MULTIPLE NORMAL MODES WITH GIVEN MINIMAL PERIOD}

This section deals with two different multiplicity results. The first result is for the special class of potentials for which different level sets are simply radial homotetic transformations of each other. These potentials, to be called similarity potentials, are of the form

$$
U(x)=F(j(x))
$$

where $F$ is a function defined on $\mathbb{R}$ and $J: \mathbb{R}^{N} \rightarrow R_{+}$is the gauge of some domain $D \subset \mathbb{R}^{N}$. More precisely, $D$ is any simply connected bounded domain in $\mathbb{R}^{N}$ with the origin in its interior and with smooth $\left(C^{2}\right)$ boundary $\partial D$. Moreover, $D$ is strictly starshaped with respect to the origin. i.e. any ray issuing from the origin intersects the boundary $\partial D$ in precisely one point, and at the point of intersection the normal to $\partial D$ is not perpendicular to the ray. Then the gauge $j$ of $D$ (the Minkowsky function) is defined as

$$
j(x):=\inf \{\lambda>0: x \in \lambda D\}, \quad x \in \mathrm{R}^{*}
$$

and $j \in C^{2}\left(\mathbb{R}^{M}\{0\}, \mathbb{R}_{+}\right), j(x)=1$ iff $x \in \partial D$. Since $j$ is homogeneous of degree 1 , it follows that $j^{\prime}(x) \cdot x=j(x)$ and $j^{\prime \prime}(x) x \cdot x=0$ for all $x \in \mathbb{R}^{N}$. Using this, it is easily verified that $U$ satisfies condition (V0)-(V2) if $D$ is a symmetric set and if $F$ satisfies the condition (V0)-(V2) for $N=1$.

A particular subset of the class of similarity potential consists of the homogeneous potentials which are of the form (4.1) with $F$ some homogeneous scalar function:

$$
F(r)=|r| \xi F(1)
$$

of some degree $\mu>0$. Note that conditions (V1)-(V2) are satisfied iff $\mu>2$ and then $U \in C^{2}\left(\mathbb{R}^{v}\right)$.

The first multiplicity result is as follows 
THEOREM 4.1. Let $U$ be a similarity potential which satisfies (V0)-(V2) and let $T^{*}$ be defined by (2.6). Then, for any $T \in\left(0, T^{*}\right)$ there exist at least $n$ distinct similar normal modes with minimal period $T$ : moreover. at least one of them corresponds via the transtormation (4) to a solution of the naturally constrained minimization problem $(2.11)$ for $\lambda=(T / 4)^{2}$.

This result is an immediate consequence of propositions 3.2.3.4 and the following lemma.

LEMMA 4.2. Let $U$ be a similarity potential of the form (4.1) with $j$ the gauge of a symmetric domain and $F$ monotonically increasing on $\mathbb{R}_{+}$. Then $U$ has at least $N$ distinct pairs $\pm e_{i}, i=$ $1, \ldots, N$ of directions of stationary increase and at least one pair are directions of maximal incrcase.

Proof. Since the gauge $j$ is an even function and $C^{2}$ on the unit sphere $S^{v-1}$, a standard result from the Ljusternik-Schnirelmann theory (see e.g. [12]) provides the existence of at least $N$ distinct pairs $\pm e_{i}, i=1, \ldots, N$ of critical points of $j$ on $S^{V-1}$. i.e. these points satisfy

$$
j^{\prime}(e)=\sigma e \text { for } \sigma=j(e)
$$

and $j$ is maximal on $S^{x-1}$ at least at one pair, say $\pm e_{1}: j\left(e_{1}\right)=\max _{e \in s^{l}-1} j(e)$.

For every $\alpha>0, U(\alpha e)=F(j(\alpha e))=F(\alpha j(e))$, and $U^{\prime}(\alpha e)=\alpha F^{\prime}(\alpha j(e)) j^{\prime}(e)$. so that $U^{\prime}(\alpha e)=\left(U^{\prime}(\alpha e) \cdot e\right) e$ for $e= \pm e_{i}, i=1, \ldots, N$. Hence all the directions $\pm e_{i}$ are directions of stationary increase. Furthermore, since $F$ is monotonically increasing. $\pm e_{1}$ are directions of maximal increase:

$$
F\left(j\left(r e_{1}\right)\right)=F\left(r j\left(e_{1}\right)\right)=F\left\{\max _{x \in r s^{\prime}-1} j(x)\right\}=\max _{x \in r S^{x-1}} F(j(x)) .
$$

The second multiplicity result of this section deals with potentials that satisfy. besides conditions (V0)-(V2), an additional condition on the geometry of the level sets. This condition states that all the level sets of $V$ can be squeezed between two balls centered at the origin that have a fixed ratio of their radii. It is interesting to note that a condition of this kind. together with a convexity assumption, has been used by Ekeland and Lasry [5] and in [7] to obtain the existence of at least $N$ distinct periodic solutions, or normal modes, which have the same value of the energy (instead of the same period), and in [9] to obtain the existence of at least $N$ distinct normal modes of the same normalized potential energy $\left(\int V(x)\right)$. This condition reads:

(V3) There exists a function $F$ defined on $\mathbb{R}$ which satisfies conditions (V0)-(V2) for $N=1$ and a constant $a \in[1,3)$ such that $V$ satisfies

$$
F(|x|) \leqslant V(x) \leqslant F(a|x|) \text { for all } x \in \mathbb{R}^{N .} .
$$

THEOREM 4.3. Suppose $V$ satisfies (V0)-(V3) and let $T^{*}$ be defined by (2.6). Then. for any $T \in\left(0 . T^{*}\right)$ there exist at least $N$ distinct normal modes $x_{T}$ of (1) with minimal period $T$. and each of these satisfies $\left|x_{T}\right| c^{0} \rightarrow x$ as $T \rightarrow 0$.

Proof. The proof is based on the following observations. For any solution $\bar{x}$ of (2) for which the corresponding normal mode does not have minimal period $T=4 \vee \lambda$, it follows from the proof of theorem 2.5, in particular from (2.13), that $\psi_{i}(\bar{x})$ satisfies $\psi_{i}(\bar{x}) \geqslant 9 c(\lambda / 9)$. Since $c$ is 
a monotonically decreasing function on $\left(0, \lambda^{*}\right)$, the interval $[c(\lambda), 9 c(\lambda / 9))$ is non-empty and consequently, any critical point $x$ of $\psi_{i}$ on $\hat{y}_{i}$ for which

$$
\psi_{i}(x)<9 c(\lambda / 9)
$$

defines a normal mode that has minimal period $T=4 \sqrt{ } \lambda$. The existence of infinitely many distinct critical points of $\psi_{i}$ on $\hat{V}_{i}$ will be a consequence of the Ljusternik-Schnirelmann theory and will be proven in part (i) under the assumptions (V0)-(V2). In part (ii) we show that because of condition (V3) at least $N$ (pairs of) critical points satisfy (4.2). each of which has $C^{0}$-norm tending to infinity as $\lambda \rightarrow 0$, which will prove the theorem.

(i) Since $V$ is even, $\psi_{i}$ is an even functional on the symmetric set $\lambda_{i}$. The presence of this $Z_{2}$-invariance allows the application of the Ljusternik-Schnirelmann theory if a certain compactness condition, the Palais-Smale condition, is verified. Under the assumptions (V0)(V2) for $V$, in particular because $\psi_{\lambda}$ satisfies $(2.15)$ on $x_{i}$, the verification of this condition is rather straightforward and we shall omit it here. Summarizing the result of this theory (see e.g. Berger [2]), let ind $(A)$ denote the genus of a symmetric. compact set $A \subset \sim_{\lambda}$, defined as (cf. $[4,10])$ ind $(A)$ is the smallest integer $k \in N \cup\{0\}$ for which there exists an odd, continuous mapping $A \rightarrow \mathbb{B}^{k} \backslash\{0\}$, and ind $(A)=x$ if no such mapping exists. The Ljusternik-Schnirelmann theory then gives the following result: if $\Sigma$ is a symmetric, compact set of $\hat{N}_{i}$ with ind $(\Sigma)=$ $n \in \mathbb{N}$ and if $b \geqslant \psi_{\lambda}(\Sigma)$, then there exist at least $n$ pairs of distinct critical points $\pm x_{i}, i=$ $1, \ldots, n$, of $\psi_{\lambda}$ on $N_{\lambda}$ which have critical value $\leqslant b$.

Since $\dot{V}_{i}$ has subsets of arbitrary large index, this shows that for any $\lambda \in\left(0, \lambda^{*}\right), \psi_{\lambda}$ has infinitely many critical points on ${ }_{i}$. Of course, not all of these solutions of (2) provide distinct normal modes.

(ii) From the summary of the Ljusternik-Schnirelmann theory above, and the introductory remarks of this proof, the theorem follows as soon as we can find some set $\Sigma$ with ind $(\Sigma)=\dot{V}$ such that

$$
\psi_{\lambda}(\Sigma)<9 c(\lambda / 9)
$$

To that end, let $V_{1}$ and $V_{a}$ be potentials defined by $V_{1}(x)=F(|x|)$, and $V_{a}(x)=F(a|x|)$. Consider for $V_{1}$ and $V_{a}$ respectively, the corresponding functionals $\psi_{i}^{1}, \psi_{i}^{a}$ and sets $\lambda \frac{1}{i}, V_{i}^{a}$ and the corresponding naturally constrained minimization problems $(2.11)$, the values of which will be denoted by $c_{1}(\lambda)$ and $c_{a}(\lambda)$ respectively.

Since $V_{1}(x) \leqslant V(x) \leqslant V_{a}(x)$ on $\mathbb{R}^{x}$, it follows that $\psi_{i}^{1} \geqslant \psi_{i} \geqslant \psi_{i}^{a}$ and, e.g. from the characterization (2.12),

$$
c_{1}(\lambda) \geqslant c(\lambda) \geqslant c_{a}(\lambda)
$$

Because of the special form of the potential $V_{a}$, the function $c_{a}$ is related in a simple way to the function $c_{1}$, explicitly

$$
c_{a}(\lambda)=a^{-2} c_{1}\left(\lambda a^{2}\right)
$$

Hence, (4.3) will certainly be satisfied if

$$
\psi_{i}(\Sigma)<9 c_{a}(\lambda / 9)=9 a^{-2} c_{1}\left(\lambda a^{2} / 9\right)
$$

We shall now construct a set $\Sigma$ for which $\psi_{\lambda}(\Sigma) \leqslant c_{1}(\lambda)$, which will imply (4.4) since $a<3$ and $c$ is monotonically decreasing on $\left(0, \lambda^{*}\right)$.

To that end, let $\lambda \in\left(0, \lambda^{*}\right)$ and let $\hat{\Sigma}$ be the solution set of the minimization problem (2.11) 
for $V_{L}$. Since $V_{\mathrm{L}}$ is rotationally symmetric, any $e \in S^{\mathrm{v}-1}$ is a direction of maximal increase for $V_{1}$. and $\hat{\Sigma}$ is given by (proposition 3.4)

$$
\hat{\Sigma}=\left\{\hat{\alpha} e: e \in S^{N-1}\right\}
$$

where $\hat{\alpha}$ is independent of $e$, and is, for any $e \in S^{v-1}$, a solution of the minimization problem (3.7) for $V_{1}$. For any $\dot{\alpha} e \in \hat{\Sigma}$, there exists a unique number $\rho>0$. depending only on $e \in S^{\nu-1}$, such that $\rho \hat{\alpha} e \in V_{i}$. Then the set

$$
\Sigma:=\left\{\rho \hat{\alpha} e \in v_{i}: e \in S^{v-1}\right\}
$$

is a compact, symmetric subset of $\gamma_{\lambda}$ and has ind $(\Sigma)=N$. Since $\psi_{\lambda} \leqslant \psi_{\lambda}^{!}$, it follows that $\psi_{\lambda}(\rho \hat{\alpha} e) \leqslant \psi_{\lambda}^{\prime}(\rho \hat{\alpha} e)$. Furthermore, because $\hat{\alpha} e$ is a solution of the minimization problem for $V_{1}$, the function $\rho \rightarrow \psi_{\lambda}^{\prime}(\rho \hat{\alpha} e)$ is maximal at $\rho=1$. Therefore

$$
\psi_{i}(\rho \hat{\alpha} e) \leqslant \psi_{i}^{\prime}(\rho \hat{\alpha} e) \leqslant \psi_{i}^{l}(\hat{\alpha} e)=c_{1}(\dot{\lambda}),
$$

which shows that $\psi_{\lambda}(\Sigma) \leqslant c_{1}(\lambda)$. This gives for any $\lambda \in\left(0, \lambda^{*}\right)$ the existence of at least $N$ pairs of critical points of $\psi_{;}$on $N_{j}$ to which their correspond at least $N$ distinct normal modes with minimal period $T=4 \sqrt{\lambda}$.

Since each of these critical points $x_{\lambda}$ belong to $\gamma_{\lambda}$, it follow's as in the proof of proposition 2.4. that $\left|x_{i}\right| C^{0} \rightarrow x$ as $\lambda \rightarrow 0$, which completes the proof.

\section{REFERENCES}

1. Ambrosetti A. \& Mavicini G.. Solutions of minimal period for a class of convex Hamiltonian systems. Math. Annaln 255. 405-421 (1981).

2. Berger M. S.. On a family of periodic solutions of Hamiltonian systems. J. diff. Eqns 10. 17-26 (1971).

3. CLARKE F. \& EKELAVD I.. Hamiltonian trajectories having prescribed minimal period, Communs pure appl. Math. 33. 103-116 (1980).

4. COFFMA.N C. V.. A minimum-maximum principle for a class of nonlinear integral equations. $J$. analvse Math. 22. $391-419$ (1969).

5. EkELA.ND I. \& LASRY J. M., On the number of periodic trajectories for a Hamiltonian flow on a convex energy surface. Ann. Math. 112, 283-319(1980).

6. Groesen E. W. C. vax, On normal modes in classical Hamiltonian systems. Int. J. Non-Linear. Hechanics 18. 5\$-70 (1983).

7. GRUESEN E. W. C. VAN, Existence of multiple normal mode trajectories on convex energy surfaces of even, classical Hamiltonian systems, J. diff. Eqns 57, 70-89 (1985).

8. GROESEN E. W. C. VAN. Applications of natural constraints in critical point theory to periodic solutions of natural Hamiltonian systems, MRC Report No. 2593 (November 1983).

9. GROESEN E. W. C. VAN, Multiple normal modes in natural Hamiltonian sỵstems; in Méthodes Topologiques en Analyse Non Linéaire (Edited by A. Granas), pp. 136-155 (1985).

10. KRASNOSELSKII M. A.. Topological Methods in the Theory of Nonlinear Integral Equations, Macmillan. New York (1964).

11. NeHARI Z., On a class of nonlinear second order differential equations. Trans. Am. math. Soc. 95. 101-123 (1960).

12. R.BINOWITZ P. H. Some aspects of nonlinear eigenvalue problems, Rocky. Mountain J. Math. 3. 161-202 (1973).

13. R.BINOWITZ P. H.. A variational method for finding periodic solutions of differential equations: in Vonlinear Evolution Equations (Edited by M. G. CRANDALL) pp. 225-251, Academic Press, New York (1975).

14. RaBrNowitz P. H. On large norm periodic solutions of some differential equations, in Ergodic Theory and Dynamical Systems. (Edited by A. K.ATOK) Vol. II. pp. 193-210. Birkhaüser, Stuttgart (1982).

15. R.iBNowitz P. H. Periodic solutions of large norm of Hamiltonian systems. J. diff. Eqns (to appear); MRC Report No. 2278. (September 1981).

16. RoSE.iBerg R. M., Normal modes of nonlinear dual-mode systems; J. appl. .Mech. 27. 263 (1960). 